\title{
Model Predictive Controller-Based Optimal Slip Ratio Control System for Distributed Driver Electric Vehicle
}

\author{
Qingxian Li, ${ }^{1}$ Liangjiang Liu ${ }^{(D)}{ }^{1}$ and Xiaofang Yuan ${ }^{2}$ \\ ${ }^{1}$ Hunan Institute of Metrology and Test, Changsha 410014, Hunan, China \\ ${ }^{2}$ College of Electrical and Information Engineering, Hunan University, Changsha 410082, Hunan, China \\ Correspondence should be addressed to Liangjiang Liu; 41326337@qq.com
}

Received 11 November 2019; Revised 20 March 2020; Accepted 27 March 2020; Published 29 April 2020

Academic Editor: Massimiliano Ferrara

Copyright (c) 2020 Qingxian Li et al. This is an open access article distributed under the Creative Commons Attribution License, which permits unrestricted use, distribution, and reproduction in any medium, provided the original work is properly cited.

The slip ratio control is an important research topic in in-wheel-motored electric vehicles (EVs). Traditional control methods are usually designed for some specified modes. Therefore, the optimal slip ratio control cannot be achieved while vehicles work under various modes. In order to achieve the optimal slip ratio control, a novel model predictive controller-based optimal slip ratio control system (MPC-OSRCS) is proposed. The MPC-OSRCS includes three parts, a road surface adhesion coefficient identifier, an operation mode recognizer, and an MPC based-optimal slip ratio control. The current working road surface is identified by the road surface adhesion coefficient identifier, and a modified recursive Bayes theorem is used to compute the matching degree between current road surfaces and reference road surfaces. The current operation state is recognized by the operation mode recognizer, and a fuzzy logic method is applied to compute the matching degree between actual operation state and reference operation modes. Then, a parallel chaos optimization algorithm (PCOA)-based MPC is used to achieve the optimal control under various operation modes and different road surfaces. The MPC-OSRCS for EV is verified on simulation platform and simulation results under various conditions to show the significant performance.

\section{Introduction}

With the development of social, the environment is getting worse [1]. Compared with traditional vehicle, EVs have great advantage in decreasing environment pollution. Therefore, more and more scholars are involved in the research of EVs $[2,3]$.

In the recent years, the slip ratio control is researched by scholars [4]. A model predictive control-based slip control was proposed [5]; the wheel slip ratio was controlled on a stable zone rather than an optimal value. Aiming at improving EV safety, a sliding mode framework control system was extended [6]. In order to achieve the multiobjective optimization control, an MPC-based slip control system for EVs was proposed [7]. A wheel slip control algorithm combined on wheel slip ratio and wheel acceleration regulation was proposed [8]. In order to achieve traction control, a decoupling state feedback controller based on the uncertain frictional coefficient was derived [9]. A robust and fast wheel slip control based on the moving sliding surface technique was proposed [10]. A model predictive controllerbased multimodel system by optimal slip ratio control was proposed [11]. The torque is assumed as a constant and the effect of the road surface adhesion coefficient is not considered in this paper. However, the EVs usually work under various operation modes and different road surfaces, while the present research studies of EVs focus on some typical modes and cannot achieve good performance under various operation modes and different road surfaces.

In order to solve this problem, a novel MPC-OSRCS is proposed in this paper. The MPC-OSRCS includes three parts, a road surface adhesion coefficient identifier, an operation mode recognizer, and an MPC based-optimal slip ratio control. The current working road surface is identified by the road surface adhesion coefficient identifier. In order to accurately describe the state of road surface, five road surface reference models are established. The matching degree between the actual road surfaces and five reference 
road surfaces is computed. A modified recursive Bayes theorem is used to calculate the matching degree. The operation mode recognizer is used to recognize the current operation state. In order to accurately describe the operation state, three operation mode reference models are established. The matching degree between the actual operation state and three operation mode reference models is computed by the fuzzy method. The PCOA [12] is used to realize the optimal design of MPC. Finally, the control output of MPC-OSRCS is computed by the weighted output of each model to achieve optimal slip ratio control under various operation states and different road surfaces.

The main contributions of this paper cover the following points. (1) The state of EV is divided into fifteen kinds of typical modes, and the reference model is established for these fifteen modes, which separately represent the link of five road surfaces and three operation modes. (2) The identifier is designed for recognizing road surface adhesion coefficient and operation mode, respectively. The output of the identifier presents the matching degree between the state of actual EV and each typical model. (3) Aiming at obtaining the control output under each state of EV, each type of matching coefficient is substituted into the controller and the weighted output of each state of EV makes up the output of MPC-OSRCS. (4) The optimal design of MPC is achieved by PCOA because of the global optimization with fast and accurate performance can be achieved by it.

\section{System Model and Problem Statement}

In order to achieve the optimal slip ratio control, a model of $\mathrm{EV}$ is established in this section, and the model of EV mainly consists of vehicle, tyre, and motor torque. Tyre model and vehicle are established according to the EV dynamics. The symbols in this paper and their physical meanings are displayed in Table 1.

2.1. Vehicle Model. The rotational movement of EV and longitudinal motion was included in the vehicle model in this paper, and the vehicle model was established based on two degree-of-freedom (2DOF) plane and shown in Figure 1. Many terms in this section are explained in detail in Rajamanis book [13]. The vehicle model can be described as follows.

Longitudinal model:

$$
m \cdot \dot{v}=F_{x f l}+F_{x f r}+F_{x r l}+F_{x r r},
$$

where $F_{x f l}, F_{x f r}, F_{x r l}$ and $F_{x r r}$ are longitudinal forces of four wheels, respectively.

Rotation movement:

$$
J \cdot \dot{\omega}=T_{e}-T_{b}-r \cdot F_{x},
$$

where $T_{b}$ is motor braking torque.

2.2. Tyre Model with Various Actuators. With the Dugoff tyre model [14], the longitudinal force of tyre is shown as follows:

$$
\begin{aligned}
F_{x} & =C_{x} \cdot \frac{\kappa}{1+\kappa} \cdot f(S), \\
f(S) & = \begin{cases}(2-S) S, & S \leq 1, \\
1, & S>1,\end{cases} \\
S & =\frac{\mu \cdot F_{z} \cdot(1+\kappa)}{2 \sqrt{\left(C_{x} \cdot \kappa\right)^{2}+\left(C_{y} \cdot \tan \alpha\right)^{2}}},
\end{aligned}
$$

where $F_{x}$ is the longitudinal force, $C_{x}$ and $C_{y}$ are the tire stiffness of longitudinal and lateral, respectively, $\mu$ is the road surface adhesion coefficient, $\kappa$ is the longitudinal slip ratio, $\alpha$ is the tire slideslips angle, and $F_{z}$ is the vertical load. The sideslip of tyre is not considered; then, the sideslip angle of tyre is considered as zero. Therefore, equation (3) can be rewritten as follows:

$$
F_{x}= \begin{cases}\mu \cdot F_{z}-\frac{\mu^{2} \cdot F_{z}^{2} \cdot(1+\kappa)}{4 \cdot C_{x} \cdot \kappa}, & \frac{\mu \cdot F_{z} \cdot(1+\kappa)}{2 \cdot C_{x} \cdot \kappa} \leq 1, \\ \frac{C_{x} \cdot \kappa}{1+\kappa}, & \frac{\mu \cdot F_{z} \cdot(1+\kappa)}{2 \cdot C_{x} \cdot \kappa}>1 .\end{cases}
$$

The value of the wheel normal force could be calculated as follows:

$$
\begin{gathered}
F_{z f l}=m\left(\frac{L_{r}}{L_{f}+L_{r}} g-\frac{h_{c g}}{L_{f}+L_{r}} a_{x}\right)\left(\frac{1}{2}-\frac{h_{c g}}{d \cdot g} a_{y}\right), \\
F_{z f r}=m\left(\frac{L_{r}}{L_{f}+L_{r}} g-\frac{h_{c g}}{L_{f}+L_{r}} a_{x}\right)\left(\frac{1}{2}+\frac{h_{c g}}{d \cdot g} a_{y}\right), \\
F_{z r l}=m\left(\frac{L_{r}}{L_{f}+L_{r}} g+\frac{h_{c g}}{L_{f}+L_{r}} a_{x}\right)\left(\frac{1}{2}-\frac{h_{c g}}{d \cdot g} a_{y}\right), \\
F_{z r r}=m\left(\frac{L_{r}}{L_{f}+L_{r}} g+\frac{h_{c g}}{L_{f}+L_{r}} a_{x}\right)\left(\frac{1}{2}+\frac{h_{c g}}{d \cdot g} a_{y}\right) .
\end{gathered}
$$

The $\kappa$ represents the wheel slip ratio, which relates to the wheel speed and the vehicle speed. The relationship can be calculated as follows:

$$
\kappa_{i}=\frac{v_{x}-\omega_{i} \cdot r}{\max \left(v_{x}, \omega_{i} \cdot r, \psi\right)}, \quad i=f l, f r, r l, r r .
$$

In order to avoid $v_{x} \longrightarrow 0 \Rightarrow \kappa_{i} \longrightarrow \infty$, the $\psi$ is set to $0.1 \mathrm{~m} / \mathrm{s}$. The longitudinal motion is only considered in this paper. In order to realize torque control, a torque balance equation is established based on vehicle characteristic for each wheel. In this case, the weight of EV is assigned to four tyres and every part equals to one quarter of the weight of the vehicle. Therefore, the single tyre model can be described as follows:

$$
\frac{1}{4} m \dot{v}=F_{x}
$$


TABLE 1: Symbols of the vehicle model.

\begin{tabular}{lcc}
\hline Definition & Symbol & $\mathrm{Unit}$ \\
\hline Vehicle mass & $m$ & $\mathrm{~kg}$ \\
Vehicle yaw moment of inertia & $I_{z}$ & $\mathrm{kgm}^{2}$ \\
Axle tread & $d$ & $\mathrm{~m}$ \\
Distance from c.g. to front and rear axle & $l_{f}, l_{r}$ & $\mathrm{~m}$ \\
Front and rear track width & $D_{f}, D_{r}$ & $\mathrm{~m}$ \\
Height of c.g. & $h$ & $\mathrm{~m}$ \\
Front and rear cornering stiffness & $C_{f}, C_{r}$ & $\mathrm{~N} /$ \\
Resistance and inductance of motor & $R, L$ & $\Omega$ \\
Flux of motor & $\rho_{f}$ & $\mathrm{~Wb}$ \\
Tyre radius & $r$ & $\mathrm{~m}$ \\
Sideslip angle in vehicle body & $\beta$ & $\mathrm{rad}$ \\
Sideslip angle of tyre & $\alpha$ & $\mathrm{rad}$ \\
Yaw rate in vehicle body & $\gamma$ & $\mathrm{rad} / \mathrm{s}$ \\
Angular velocity of tyre & $\omega$ & $\mathrm{rad} / \mathrm{s}$ \\
Pitch rate in vehicle body & $\rho$ & $\mathrm{rad} / \mathrm{s}$ \\
Front wheel steering angle from controller and & $\delta_{f}, \delta$ & $\mathrm{rad}$ \\
driver & $\kappa$ & - \\
Tyre longitudinal slip ratio & $F_{y f}, F_{y r}$ & $\mathrm{~N}$ \\
Later force of front or rear tyres & $J$ & $\mathrm{kgm}$ \\
Moment of inertia of each wheel & $a_{x}, a_{y}$ & $\mathrm{~m} / \mathrm{s}^{2}$ \\
Longitudinal or lateral acceleration & $\mu$ & - \\
Friction coefficient of road & $T_{e}$ & $\mathrm{Nm}$ \\
Longitudinal torque of each wheel & $T_{t}$ & $\mathrm{Nm}$ \\
Total drive torque from driver pedal command & $\widehat{M}_{z}$ & $\mathrm{Nm}$ \\
Additional yaw moment of each wheel & $K_{a}, K_{b}$ & - \\
Fitting coefficients & &
\end{tabular}

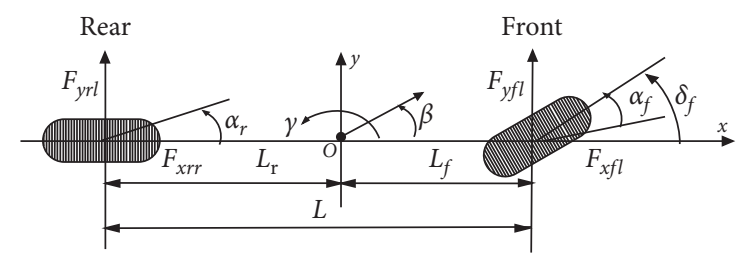

FIgURE 1: 2DOF vehicle model.

Driving motion: the EV works under uniform speed or acceleration operation mode while $r \cdot \omega>v$. According to equations (1)-(5), (11), and (12), the $\kappa$ can be described as follows, where $v_{x}=v$ :

$$
\dot{\kappa}=\left(-\frac{r^{2}}{J v}-\frac{\kappa+1}{(1 / 4) m v}\right) \cdot F_{x}+\frac{r}{J v}\left(T_{e}-T_{b}\right) .
$$

Braking motion: the EV works under braking operation mode while $v>r \cdot \omega$. According to equations (1)-(5), (11), and (12), the $\kappa$ can be described as follows:

$$
\dot{\kappa}=\left(\frac{r^{2}}{J v}+\frac{\kappa+1}{(1 / 4) m v}\right) \cdot F_{x}-\frac{r}{J v}\left(T_{e}-T_{b}\right) .
$$

According to equations (6) and (12), $(\mathrm{d} \mu / \mathrm{d} \kappa)$ is shown as follows [15]:

$$
\frac{\mathrm{d} \mu}{\mathrm{d} \kappa}=\frac{\left((1 / 4) \cdot m \cdot\left(d^{2} v / d t^{2}\right)+\left(F_{z} \cdot \mu^{2} / 4 \cdot C_{x} \cdot \kappa^{2}\right)\right)}{\left(1-\left(2 \cdot F_{z} \cdot \mu / 4 \cdot C_{x}\right)+\left(F_{z} \cdot \mu / 4 \cdot C_{x} \cdot \kappa\right)\right)} .
$$

2.3. Control Problem Formulation. In order to realize the optimal slip ratio control, some problems should be considered in this paper:

(1) Various operation modes and different road surfaces problem: EVs usually work under different operation modes and various road surfaces. A mount of research studies show that the slip ratio is related to the adhesion coefficient and operation mode [16-18]. Therefore, different operation modes and various adhesion coefficients should be considered.

(2) Constraints problem: In order to keep the EV in a stability state, all the control parameters should be kept at constraint range. The $\kappa$ should be limited within the stable slip ratio range for each road surface. $\kappa_{p}$ is the optimal for each road surface:

$$
-\kappa_{\max } \leq \kappa \leq \kappa_{\max }, \quad \kappa_{\max }=\kappa_{p} .
$$

In addition, the motor torque command $T$ should be limit within the maximum motor output torque $T_{\max }$ [7], and it is shown as follows:

$$
-T_{\max } \leq T \leq T_{\max }
$$

\section{Design of Control System}

In order to realize the optimal slip ratio control when $\mathrm{EV}$ works on various operation modes and different road surfaces, a novel MPC-OSRCS is proposed in this paper. Figure 2 shows the control structure of MPC-OSRCS. In the MPC-OSRCS, two recognizers are established according to the characteristics of EV, and the optimal slip ratio control is realized by a MPC under various operation modes and different road surfaces. The MPC-OSRCSs can be explained as follows. (1) The road surface adhesion coefficient identifier is used to identify the current working road surface. It includes five reference models and represents five typical road surfaces, respectively. Each reference model can accurately represent the state of road surfaces. A modified recursive Bayes theorem is used to calculate the matching degree between the actual road surfaces and five reference road surfaces. (2) The operation mode recognizer is used to recognize the current operation state. In order to accurately describe the state of operation state, three operation mode reference models are established. A fuzzy logic method is used to calculate the matching degree between the actual operation state and three reference operation modes. (3) The optimal slip ratio control under various operation modes and different road surfaces is realized by a MPC, and the optimal design of MPC is achieved by PCOA. (4) In order to obtain the control output under each state of EV, each type of matching coefficient is substituted into the controller and the weighted output of each state of EV makes up the output of MPC-OSRCS.

\subsection{The identifier of Road Surfaces Adhesion Coefficient.}




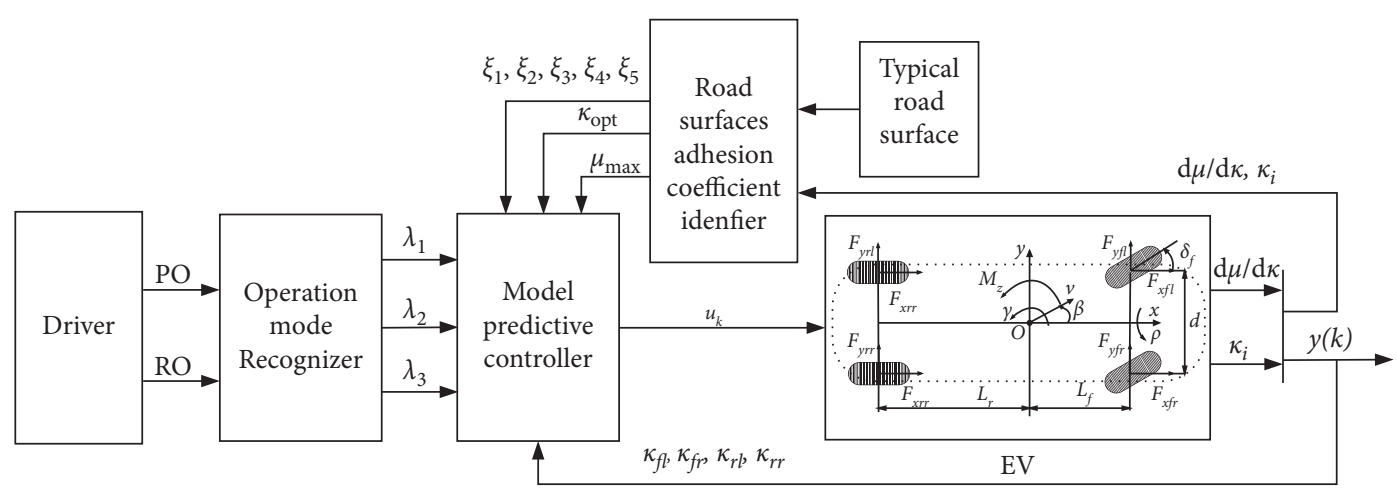

FIgURE 2: The structure of MPC-OSRCS for EV.

The slip ratio $\kappa$ is related to the road surface adhesion coefficient $\mu$, different road surfaces have various characteristic between adhesion coefficient $\mu$, and slip ratio $\kappa . \mu-\kappa$ function curve equation is proposed by Zhang et al. [19]; $\mu-\kappa$ function is easy and accurate to describe the mathematical relationship between the slip ratio $\kappa$ of tyre based on various roads and the adhesion coefficient $\mu$ as follows:

$$
\begin{gathered}
\mu(\kappa)=c_{1} \cdot\left(1-\exp \left(-c_{2} \cdot \kappa\right)\right)-c_{3} \cdot \kappa, \\
\frac{\mathrm{d} \mu}{\mathrm{d} \kappa}=c_{1} \cdot c_{2} \cdot \exp \left(-c_{2} \cdot \kappa\right)-c_{3},
\end{gathered}
$$

where $c_{1}, c_{2}$, and $c_{3}$ are the fitting coefficients for various road surfaces according to via experiment research. The parameters of five typical road surface are shown in Table 2.

In the design of road surface adhesion coefficient identifier, it is difficult to compute the matching degree between the actual road surfaces and five reference road surfaces. The switching idea between two operation modes has been introduced in many literature studies [20-22]. Based on the same method, this theory can also be used to identify road conditions.

A modified recursive Bayes theorem is used to compute the weight coefficient $\xi_{j}$, where $\xi_{j}$ represents the matching degree between the actual road surfaces and each reference road surface. The former relative error is applied to the calculation of $\xi_{j}$ and then the smooth switching can be achieved. The posterior probability is evaluated by a modified recursive Bayes theorem for the $j$ th model (or value) at the $k$ th time instant and can be described as follows:

$$
P_{j, k}=\frac{H \cdot \exp \left(-(1 / 2) \varepsilon_{j, k}^{T} \cdot G \cdot \varepsilon_{j, k}\right) \cdot P_{j, k-1}}{\sum_{j=1}^{5} H \cdot \exp \left(-(1 / 2) \varepsilon_{j, k}^{T} \cdot G \cdot \varepsilon_{j, k}\right) \cdot P_{j, k-1}}, \quad j=1,2,3,4,5,
$$

where $\varepsilon_{j, k}=\left(y_{m}(k)-y_{j}(k)\right) / y_{m}(k)$ represents the relative error between the actual output state $y_{j}(k)$ and the reference value $y_{m}(k) . P_{j}(k)$ represents the posterior probability in the $j$ th linearized model at $k$ moment. $H=\left[\begin{array}{cccc}h_{1} & 0 & 0 & 0 \\ 0 & h_{2} & 0 & 0 \\ 0 & 0 & h_{3} & 0 \\ 0 & 0 & 0 & h_{4}\end{array}\right]$ represents the weight matrix between state variable $k_{j}$,
TABLE 2: Parameters of road surface adhesion coefficient.

\begin{tabular}{lccc}
\hline Road surface & $c_{1}$ & $c_{2}$ & $c_{3}$ \\
\hline Dry asphalt & 1.28 & 23.99 & 0.52 \\
Wet asphalt & 0.86 & 33.82 & 0.35 \\
Wet pebbles & 0.40 & 33.71 & 0.12 \\
Snow & 0.19 & 94.13 & 0.06 \\
Ice & 0.05 & 306.4 & 0.001 \\
\hline
\end{tabular}

$h_{e}(e=1,2,3,4)$ represents the influence factor and relates to control performance, and $G$ represents a time invariant weight matrix and usually is selected to a diagonal. According to the normal distribution, $G$ represents the inverse matrix of the residual covariance. The higher parameter of $G$ means that the residual variance is small and the confidence in the residual of each model is greater. The higher the value of the element of $G$ is, the ability of rejection of model with large residual is more stronger. The weight coefficient of road surfaces $\xi_{j}$ is described as follows:

$$
\xi_{j}=\frac{P_{j, k}}{\sum_{j=1}^{3} P_{j, k}}, \quad j=1,2,3,4,5 .
$$
is 1 :

Here, $\xi_{j}$ is a value between 0 and 1 . The summation of $\xi_{j}$

$$
\sum_{j=1}^{5} \xi_{j}=1
$$

3.2. The Recognizer of Operation Modes. The T-S fuzzy controller is applied to recognize various operation modes in this paper, and it is proposed by the scholars Takagi and Sugeno in 1985. The actual of pedal opening (PO) and the change rate of pedal opening (RO) are the inputs of the T-S fuzzy controller. If the EV is under driving modes, the pedal opening is defined as positive. Otherwise, the PO is defined as negative. The $\mathrm{PO}$ and $\mathrm{RO}$ are different under various operation modes. The output of the T-S fuzzy controller is membership value $\eta_{i}, i=1,2,3$, which represents three typical operation modes: acceleration, uniform speed, and deceleration.

The values of $\mathrm{PO}$ and $\mathrm{RO}$ are limited within $[-100,100]$. The value of $\mathrm{PO}$ is defined as seven kinds of states, there are zero $(\mathrm{Z})$, 
very small (VS), small (S), medium (M), big (B), very big (VB), and great $(\mathrm{G})$, respectively. At the same time, the value of $\mathrm{RO}$ is defined as five kinds of states, and there are very small (VS), small (S), medium (M), big (B), and very big (VB), respectively. The value of $\eta$ is defined as four kinds of states, and there are very small (VS), small (S), medium (M), and big (B). The membership functions for PO and RO are set, as shown in Figure 3.

Table 3 shows the rule set in the fuzzy inference system (FIS). The rule set includes $7 * 5=35$ rules.

Rule1: if PO is " $\mathrm{Z}$," $\mathrm{RO}$ is "VS," then $\eta_{1}$ is "B," $\eta_{2}$ is "S," and $\eta_{3}$ is "S."

.

Rule35: if $\mathrm{PO}$ is "G," RO is "VB," then $\eta_{1}$ is " $\mathrm{S}$," $\eta_{2}$ is " $\mathrm{S}$," and $\eta_{3}$ is "M."

In this way, the whole rules can be obtained.

Here, $\sum_{i=1}^{3} \lambda_{i}=1$ and $\lambda_{i} \in[0,1]$, where $i=1,2,3$ Normalization processing is carried out to calculate the weight coefficient $\lambda_{i}$ as follows:

$$
\lambda_{i}=\frac{\eta_{i}}{\sum_{j=1}^{3} \eta_{i}},
$$

where $\eta_{i}$ is membership value of three typical operation modes.

3.3. Design of the MPC. The optimal and constraint problem are efficiently solved by MPC. MPC is selected to optimum $\kappa$ for each operation mode and each road surface in this paper. MPC has a great advantage to achieve satisfy diverse and even conflicting requirements of vehicles [23] as its characteristic. In addition, the effect of model mismatch and unmeasured disturbance can be attenuated by MPC [24, 25]. Moreover, the effect of switching between various road surfaces and different operation modes can be attenuated by the weighted output of each MPC.

3.3.1. Model Predictive Control Law. $x$ represents state vector, $u$ represents control vector, and $y$ represents output vector, and they can be expressed as follows:

$$
\begin{gathered}
x=\left[\begin{array}{c}
\kappa_{f l} \\
\kappa_{f r} \\
\kappa_{r l} \\
\kappa_{r r}
\end{array}\right], \\
u=\left[\begin{array}{c}
T_{f l} \\
T_{f r} \\
T_{r l} \\
T_{r r}
\end{array}\right], \\
y=\left[\begin{array}{c}
\kappa_{f l} \\
\kappa_{f r} \\
\kappa_{r l} \\
\kappa_{r r}
\end{array}\right] .
\end{gathered}
$$

In order to achieve the optimal MPC design, time statespace models in equations (12)-(13) are discrete by the Euler method. $k$ represents the sample time and is defined as $k=\operatorname{int}\left(t / T_{s}\right)$, where running time is defined as $t$ and fixed step size is defined as $T_{s}$. At sample time $k$, the predictive state is calculated as follows:

$$
\begin{aligned}
x(k+1) & =f^{k}((x+1), u(k)) \cdot T_{s}+x(k), \\
y(k) & =C \cdot x(k) .
\end{aligned}
$$

Then, the discretization of the state space model of equation (12) is described as equation(27), and the discretization of state space of equation (13) is described as equation (28):

$$
\begin{aligned}
x_{e}(k+1)= & \left(-\frac{r^{2}}{J v}-\frac{x_{e}(k)+1}{(1 / 4) m v}\right) \cdot F_{x} \cdot T_{s} \cdot x_{e}(k) \\
& +\frac{r}{J v} \cdot u_{e}(k) \cdot T_{s} \cdot x_{e}(k)+x_{e}(k), \quad e=1,2,3,4, \\
x_{e}(k+1)= & \left(\left(\left(\frac{r^{2}}{J v}+\frac{x_{e}(k)+1}{(1 / 4) m v}\right) \cdot F_{x}-\frac{r}{J v} \cdot u_{e}(k)\right) \cdot T_{s}+1\right) \\
& \cdot x_{e}(k) .
\end{aligned}
$$

According to the principle of MPC, $p$ represents a predictive horizon and $m$ represents a control horizon. $m=$ $p=6$ in this paper. $U(k)$ represents the optimization vectors and $Y(k)$ represents the predictive control output. They can be expressed as follows:

$$
\begin{aligned}
& U(k)=\left[\begin{array}{c}
u(k \mid k) \\
u(k+1 \mid k) \\
\cdot \\
u(k+m-1 \mid k)
\end{array}\right]_{m \times 1}, \\
& Y(k)=\left[\begin{array}{c}
y(k \mid k) \\
y(k+1 \mid k) \\
\cdot \\
y(k+m \mid k)
\end{array}\right]_{p \times 1},
\end{aligned}
$$

where $U(k)$ is defined as an array of control input $u$ and $Y(k)$ represents the output vector from sampling time $k$ to sampling time $k+i$. Moreover, $R(k)$ represents reference sequence. $u(k)$ is defined as the control input change and can be calculated by $\Delta u(k)=u(k)-u(k-1)$. They can be defined as follows:

$$
\begin{aligned}
R(k) & =\left[\begin{array}{c}
r(k) \\
r(k) \\
\cdot \\
r(k)
\end{array}\right]_{p \times 1}, \\
\Delta U(k) & =\left[\begin{array}{c}
\Delta u(k \mid k) \\
\Delta u(k+p \mid k) \\
\cdot \\
\Delta u(k+m-1 \mid k)
\end{array}\right]_{m \times 1} .
\end{aligned}
$$

According to the principle of MPC, the relationship of $u(k)$ and $U(k)$ can be described as follows: 


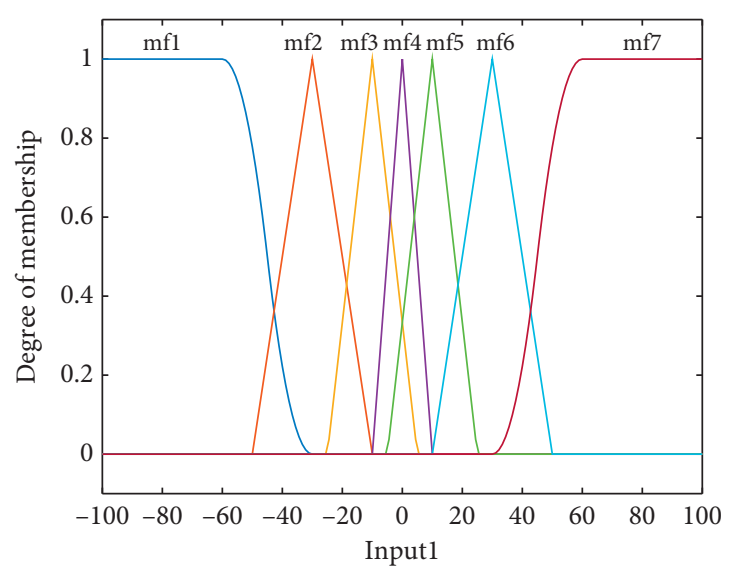

(a)

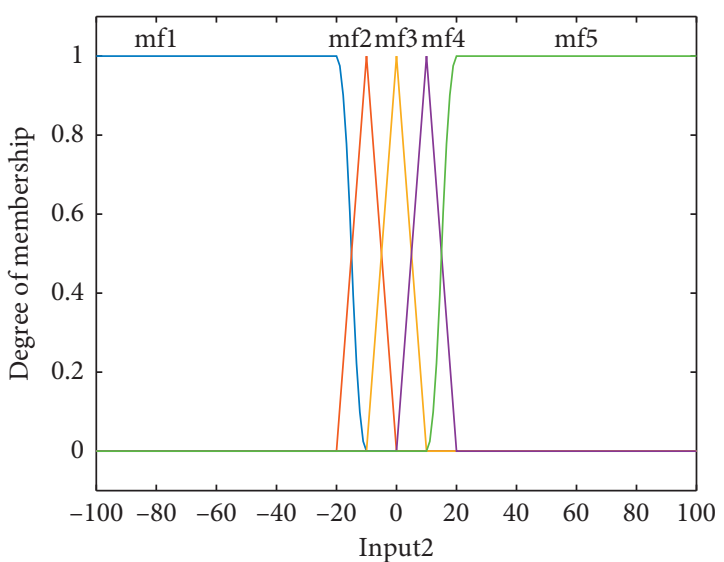

(b)

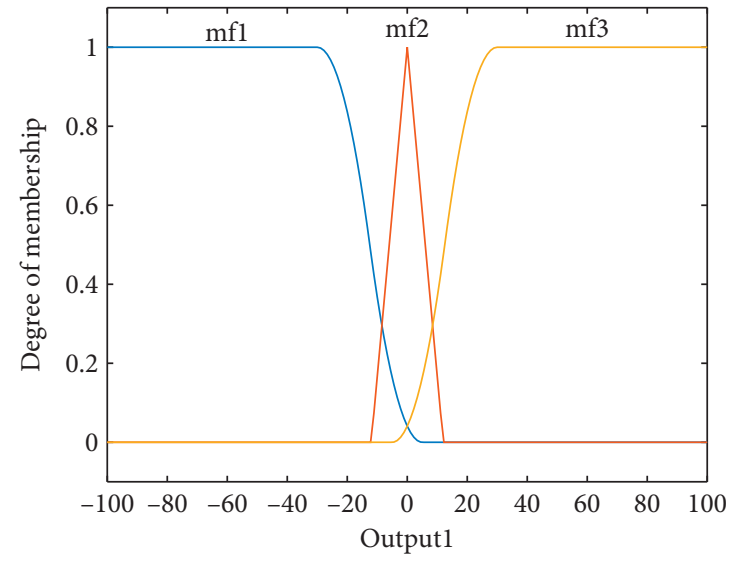

(c)

Figure 3: The membership functions. (a) PO. (b) RO. (c) $\eta_{i}$.

$$
u(k)=[1,0, \ldots, 0] \cdot U(k)
$$

where $u(k)$ represents optimal vectors, which is used to compute the optimal control action of the EV system.

3.3.2. Design of the Objective Function. In order to realize the optimal control, the multiple objective function of the MPC is established and it is achieved by minimization of this multiple objective function. The optimization function includes two parts. One is the motor torque $T$; it includes drive torque $T_{e}$ and brake torques $T_{b}$; the other is slip ratio $\kappa$ of the four wheels. The cost function is described as follows:

(1) The main importance is to make the actual slip ratio $\kappa$ track the optimal slip ratio $\kappa_{p}$, whatever the conditions of EV are. Therefore, the first cost function can be described as follows:

$$
J_{1}=\|Y(k)-R(k)\|_{Q}^{2}
$$

where $Q$ represents a positive weight factors and can be used to adjust the performance of tracking.
(2) In order to save energy, the sum of torque command squares should be controlled as small as possible. Therefore, the second cost function can be calculated as follows:

$$
J_{2}=\|U(k)\|_{R}^{2}
$$

where $R$ represents a positive weight factors and can be used to adjust the performance of $T$.

(3) Aiming at achieving the optimal slip ratio control, the actual slip ratio $\kappa$ needs to track the optimal slip ratio $\kappa_{p}$ as accurate as possible. Hence, the third cost function can be calculated as follows:

$$
J_{3}=\left\|\kappa(k)-\kappa_{p}(k)\right\|_{F}^{2}
$$

(4) The tyre longitudinal performance is related to $T$. Therefore, in order to ensure the longitudinal stability of $\mathrm{EV}, T$ is limited within $T_{\max }$, and the forth cost function can be calculated as follows: 
TABLE 3: Fuzzy logic rule set.

\begin{tabular}{|c|c|c|c|c|}
\hline $\mathrm{PO}$ & RO & $\eta_{1}$ & $\eta_{2}$ & $\eta_{3}$ \\
\hline Z & VS & B & $S$ & $S$ \\
\hline Z & $\mathrm{S}$ & B & S & $S$ \\
\hline Z & $\mathrm{M}$ & B & $S$ & $S$ \\
\hline Z & B & B & $S$ & $S$ \\
\hline Z & VB & B & S & S \\
\hline VS & VS & B & $S$ & $S$ \\
\hline VS & $\mathrm{S}$ & B & $S$ & S \\
\hline VS & $\mathrm{M}$ & B & $S$ & $S$ \\
\hline VS & B & B & $S$ & $S$ \\
\hline VS & VB & B & $S$ & $S$ \\
\hline S & VS & B & $\mathrm{S}$ & S \\
\hline$S$ & $S$ & B & $\mathrm{M}$ & $S$ \\
\hline$S$ & $\mathrm{M}$ & B & $\mathrm{M}$ & $S$ \\
\hline$S$ & B & B & $\mathrm{M}$ & $S$ \\
\hline S & VB & B & $S$ & $S$ \\
\hline $\mathrm{M}$ & VS & $\mathrm{M}$ & B & $S$ \\
\hline $\mathrm{M}$ & $\mathrm{S}$ & $\mathrm{M}$ & B & $S$ \\
\hline $\mathrm{M}$ & $\mathrm{M}$ & $\mathrm{M}$ & B & $\mathrm{M}$ \\
\hline $\mathrm{M}$ & B & $S$ & $\mathrm{M}$ & B \\
\hline $\mathrm{M}$ & VB & $S$ & M & B \\
\hline B & VS & B & $\mathrm{M}$ & $S$ \\
\hline B & $S$ & $\mathrm{M}$ & B & $S$ \\
\hline B & $\mathrm{M}$ & $\mathrm{M}$ & B & $\mathrm{M}$ \\
\hline B & B & $\mathrm{S}$ & $\mathrm{M}$ & B \\
\hline B & VB & $S$ & $S$ & B \\
\hline VB & VS & B & $S$ & S \\
\hline VB & $S$ & $\mathrm{~S}$ & B & $S$ \\
\hline VB & $\mathrm{M}$ & $\mathrm{M}$ & B & $\mathrm{M}$ \\
\hline VB & B & $S$ & $\mathrm{M}$ & B \\
\hline VB & VB & S & $\mathrm{S}$ & B \\
\hline G & VS & B & $S$ & S \\
\hline G & $S$ & $\mathrm{M}$ & B & $S$ \\
\hline $\mathrm{G}$ & $\mathrm{M}$ & $S$ & B & $S$ \\
\hline $\mathrm{G}$ & B & $S$ & $\mathrm{M}$ & B \\
\hline $\mathrm{G}$ & VB & $S$ & $S$ & B \\
\hline
\end{tabular}

$$
J_{4}=\left\|U(k)-T_{\max }\right\|_{G}^{2}
$$

Therefore, the total cost function can be defined as follows:

$$
J=J_{1}+J_{2}+J_{3}+J_{4}
$$

3.3.3. The Process of Optimal Design. The optimal design is realized by a novel PCOA, and we have carried out some works about PCOA. In addition, the PCOA has great advantage to realize global optimum. Objective function in the PCOA is described in equation (30) and it can be rewritten as follows:

$$
\min f(X)=f\left(x_{1}, x_{2}, x_{3}, x_{4}\right), x_{i} \in\left[L_{i}, P_{i}\right],
$$

where $X=f\left(x_{1}, x_{2}, x_{3}, x_{4}\right) \in W^{4}$ is a vector in the 4 -dimensional decision variables space, $x_{i}$ represents different variables parameters and $x_{i} \in\left[L_{i}, P_{i}\right], L_{i}$ represents the lower bound of the $i$ th variable, and $P_{i}$ represents the upper bound of the $i$ th variable. Then, PCOA evolves a stochastic population of $N$ candidate individuals with 4-dimensional parameter vectors; the candidate individuals will experience twice carrier wave mechanism. The different chaotic traces are obtained by the first carrier wave; the search precision is enhanced by the second carrier wave.

3.4. The Control Output of MPC-OSRCS. As described above, the weight coefficient of different reference models and the output of each MPC are calculated. At each sampling point, the control output of MPC-OSRCS is constituted by the output of each MPC $u_{i \cdot j}(k)$ and its weight coefficient. Therefore, the control output of MPC-OSRCS can be described as follows:

$$
u(k)=\sum_{i=1}^{3} \sum_{j=1}^{5} \lambda_{i} \cdot \xi_{j} \cdot u_{i \cdot j}(k),
$$

where $u(k)$ represents the control output of MPC-OSRCS, the $u_{i \cdot j}(k)$ represents the $i \cdot j$ th reference model, and $\lambda_{i}$ and $\xi_{j}$ are the weight coefficients.

\section{Simulation and Analysis}

The computer simulation is used to verify the control performance of the proposed MPC-OSRCS. The simulation is based on an $8 \mathrm{DOF}$ model simulation platform, as shown in Figure 4.

The simulation is carried under three operation modes and five road surfaces. Aiming at verifying the advantage of the MPC-OSRCS, the performance of the MPC-OSRCS is compared with the tractional MPC [18] and the model predictive controller-based multimodel control system (MPCMMCS). The simulation time is set $5 \mathrm{~s}$, and the fixed step size $T_{s}$ is chosen as $5 \mathrm{~ms}$. The simulation parameters are set as follows: $Q=\operatorname{diag}\left(10^{4}, 10^{4}, 10^{4}, 10^{4}\right), R=\operatorname{diag}(10,10,10,10)$, $F=\operatorname{diag}\left(10^{4}, 10^{4}, 10^{4}, 10^{4}\right), S=\operatorname{diag}\left(10^{4}, 10^{4}, 10^{4}, 10^{4}\right), m=$ $1359.8 \mathrm{~kg}, I_{z}=1992.54 \mathrm{kgm}^{2}, L_{f}=1.0628 \mathrm{~m}, L_{r}=1.4852 \mathrm{~m}$, $D_{f}=1.0628 \mathrm{~m}, \quad C_{y}=23540(\mathrm{~N} / \mathrm{rad}), \quad C_{x}=23101(\mathrm{~N} / \mathrm{rad})$, $J=0.3534 \mathrm{kgm}^{3}, L=0.05 \mathrm{Hm}, \rho_{f}=0.1 \mathrm{~Wb}, r=0.29 \mathrm{~m}$, and $h=0.512 \mathrm{~m}$.

4.1. Test 1 . The EV works on straight running in test 1 , and the start speed of $0 \mathrm{~km} / \mathrm{h}$. The change of vehicle velocity and the change of road surfaces are considered in this simulation. The vehicle velocity $v$ increases from $10 \mathrm{~km} / \mathrm{h}$ to $80 \mathrm{~km} / \mathrm{h}$ during $0-1.75 \mathrm{~s}$, keeps uniform speed during $1.75-3.25 \mathrm{~s}$, and decreases from $80 \mathrm{~km} / \mathrm{h}$ to $10 \mathrm{~km} / \mathrm{h}$ during $3.25-5 \mathrm{~s}$. The road surfaces are wet asphalt during $0-0.75 \mathrm{~s}$, the road surface is wet pebbles during $0.75-1.5 \mathrm{~s}$, the road surface is dry asphalt during $1.5-2.5 \mathrm{~s}$, the road surface is ice during $2.5-4 \mathrm{~s}$, the road surface is snow during $4-5 \mathrm{~s}$. The traditional method means that only a MPC under various operation modes and different road surfaces [17]. The input variables of the traditional MPC are four motor torques from $\mathrm{EV}$, and the output variables are actual state values of the actual state value of $\kappa_{f l}, \kappa_{f r}, \kappa_{r l}$, and $\kappa_{r r}$.

4.1.1. The Performance of Traditional MPC. The simulation results of the traditional MPC are shown in Figure 5. 


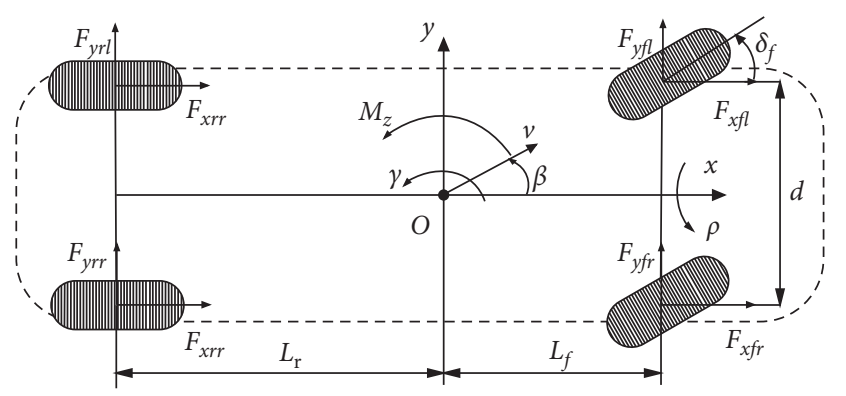

Figure 4: 8DOF vehicle model.

As shown in Figure 5(a), the slip ratio is very big at the beginning, even close to 1 . With the increase of velocity, the slip ratio is kept on a steady range. It is clearly shown in Figure 5(a) that the actual slip ratio has not changed following the road surfaces changes; in other words, the slip ratio is only a steady value rather than an optimal value. It is proved that the traditional MPC cannot be suitable to the multimodes. Therefore, the traditional MPC is difficult to achieve good performance and realize the optimal slip ratio control in actual working.

We can see from Figure 5(b) that the motor torques of four wheels $T_{e}(e=1,2,3,4)$ are appropriate and equal to $80 \mathrm{~N}$. The motor torques of four wheels is not following the road surface changes, whatever the road surface is wet asphalt or snowy. The required motor torques of four wheels are different while the road surfaces changes. In the switching process between various operation modes and different road surfaces, the oscillatory behavior may occur.

From the results of simulation, we can find that traditional MPC cannot realize the optimal slip ratio control while EV switches on various operation modes and different road surfaces; then, it is difficult to ensure the longitudinal stability of EVs.

4.1.2. The Performance of MPC-MMCS. The simulation results of MPC-MMCS are shown in Figure 6. As Figure 6(a) shows, the optimal slip rate follows the road surfaces change in electric vehicle operation. However, the optimal slip rate cannot achieve when the road surfaces changes. It is easy to find in Figure 6(b) that $T$ is also kept within a stable range.

The wheel velocity is displayed in Figure 6(c), the smoothly switching can be achieved when the EV works under different operation modes and various surfaces road surfaces. In Figure $6(\mathrm{~d})$, the $\lambda_{i}$ can accurately reflect the operation modes of EV.

In conclusion, the MPC-MMCS can successfully recognize the operation modes of EV. Yet the optimal slip rate cannot achieve when the EV works under different road surfaces.

4.1.3. The Performance of MPC-OSRCS. The simulation results of MPC-OSRCS are shown in Figure 7. Note that the weight coefficient $\lambda_{i}$ and $\xi_{j}$ are calculated by the fuzzy method and a modified recursive Bayes theorem. They represent the matching degree between the state of actual EV and three reference operation modes and between the actual road surfaces and reference road surfaces, respectively.

The biggest challenges in the proposed MPC-OSRCS are the optimal slip ratio control under different operation modes and various road surfaces. As shown in Figure 7(a) that the optimal wheel ratio slip can be achieved no matter what the state of actual $\mathrm{EV}$, in addition, the optimal slip rate follows the road surfaces change in electric vehicle operation. While the EV has switched different road surfaces, the optimal slip ratio also can smoothly achieved. We can see from the picture that the optimal slip ratio control can be realizes by proposed MPC-OSRCS when EV switches on various operation modes and different road surfaces.

Figure 7(b) illustrates the wheel velocity $r \cdot \omega$. We can see from Figure 6(b) that EV runs smoothly when it switches on various operation modes and different roads surfaces. We can see from Figures 7(c) and 7(d) that the $\lambda_{i}$ represents the matching degree between the state of actual EV and each operation modes, and the $\xi_{j}$ represents the matching degree between the actual roads surfaces and the reference road surfaces. As described above, the matching degree is almost identical to operation conditions.

We can see from Figure 7(e) that $T$ changes smoothly while the state of EV changing. Whether the change of operation modes or the road surfaces, the $T$ is also kept within a stable range $T=240$. The difference between motor torques of four wheels is small and the comfortable of EV is well.

Compared with the traditional MPC, the proposed MPC-OSRCS can realize the smooth switching and optimal slip ratio control whatever the running state of EV. The simulation clearly proves that the proposed MPC-OSRCS can be suitable for different operation modes and various road surfaces and all can achieved smooth switching and optimal slip ratio control.

4.2. Test 2. The EV also is tested on various operation modes and different road surfaces in test 2 . The starting speed of EV is $85 \mathrm{~km} / \mathrm{h}$ and the straight running only is considered. The state of EV is deceleration, its velocity decreases from $80 \mathrm{~km} / \mathrm{h}$ to $10 \mathrm{~km} / \mathrm{h}$ during $0-1.75 \mathrm{~s}$, and then the EV keeps the speed unchanged. At this time, the state of EV is uniform speed during $1.75-3.25 \mathrm{~s}$. At the next time, the EV is acceleration and the velocity of EV increase from $10 \mathrm{~km} / \mathrm{h}$ to $80 \mathrm{~km} / \mathrm{h}$ during $3.25-5 \mathrm{~s}$. At the same time, the road surfaces are also changed over time. At the beginning, the EV is driving on the ice road surface. After $0.75 \mathrm{~s}$, the road surfaces are changed to snow road surface. After $0.75 \mathrm{~s}$, the road surfaces are changed to wet pebbles road surface. After $1 \mathrm{~s}$, the road surfaces are changed to wet asphalt road surface. After $1.5 \mathrm{~s}$, the road surfaces are changed to dry asphalt road surface.

4.2.1. The Performance of Traditional MPC. The simulation results of the traditional MPC are shown in Figure 8.

As shown in Figure 8(a), we can see that the slip ratio is mainly affected by operation modes and the road surfaces 


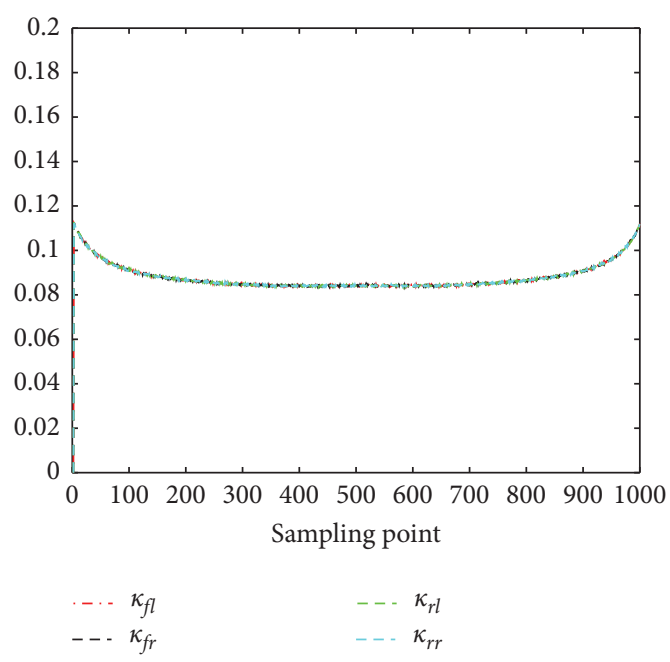

(a)

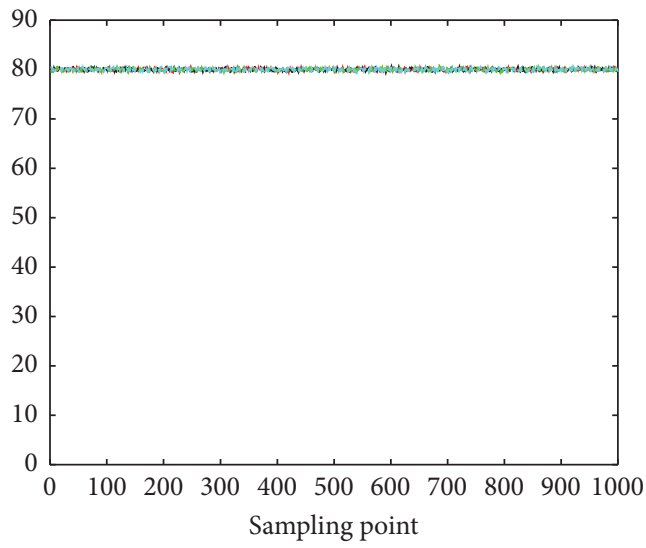
$\ldots T_{f l}$
-.- $T_{f r}$
$\ldots T_{r l}$
$T_{r r}$

(b)

Figure 5: The simulation results of conventional MPC on test 1. (a) Tire longitude slip ratio $\kappa$. (b) Distribution of motor torques.

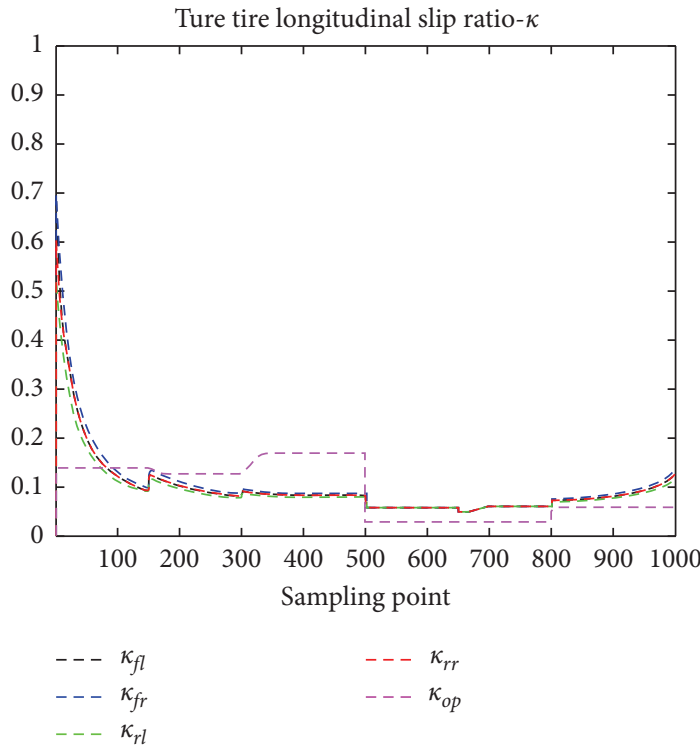

(a)
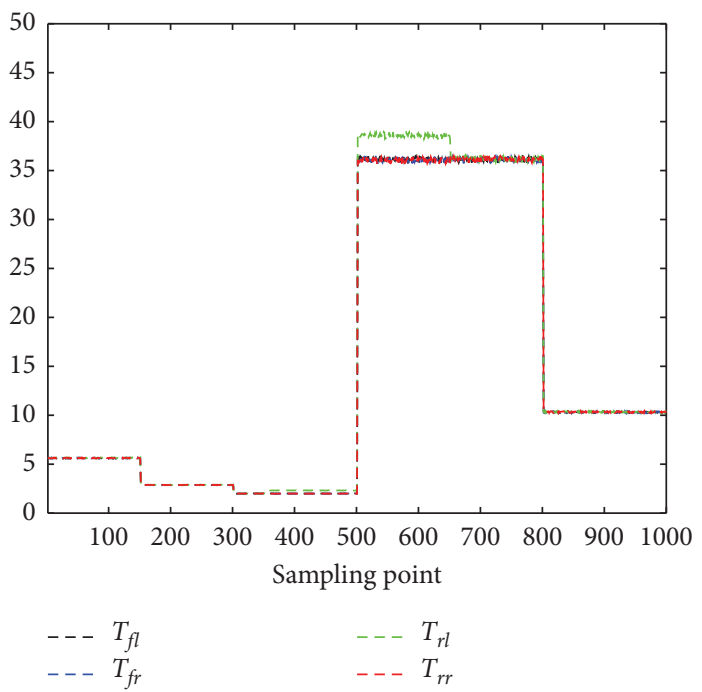

Figure 6: Continued.

(b) 


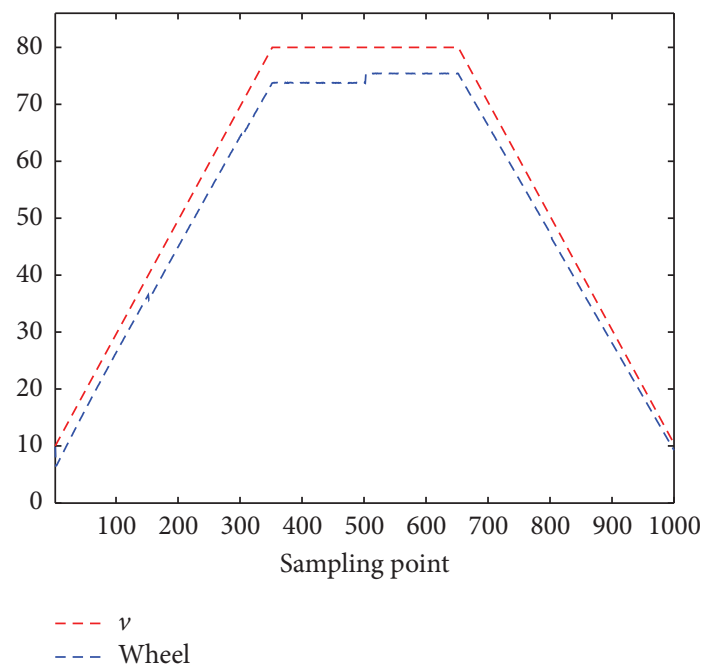

(c)

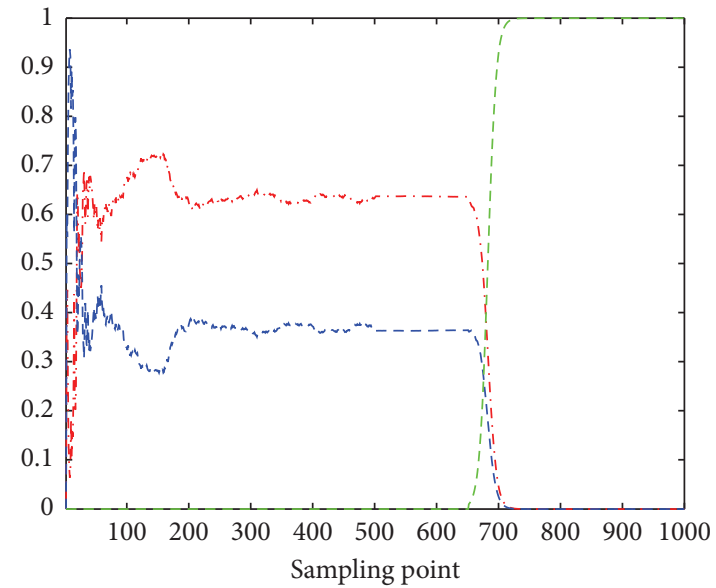

-.. Weight of model1 _ - - Weight of model3

- _- Weight of model2

(d)

Figure 6: The simulation results of MPC-MMCS on test 1. (a) Tire longitude slip ratio $\kappa$. (b) Distribution of motor torques. (c) Vehicle velocity. (d) Weight of operation modes.

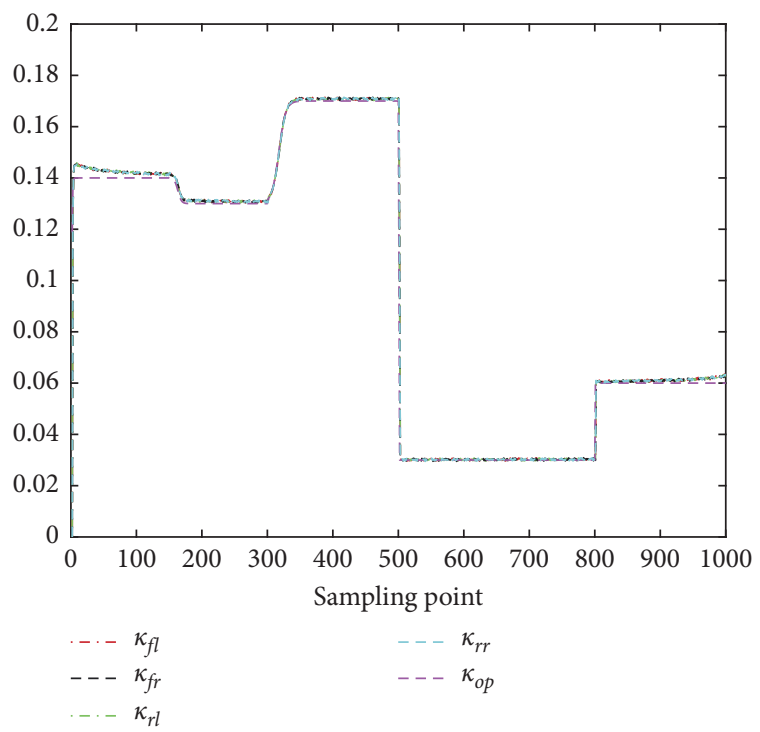

(a)

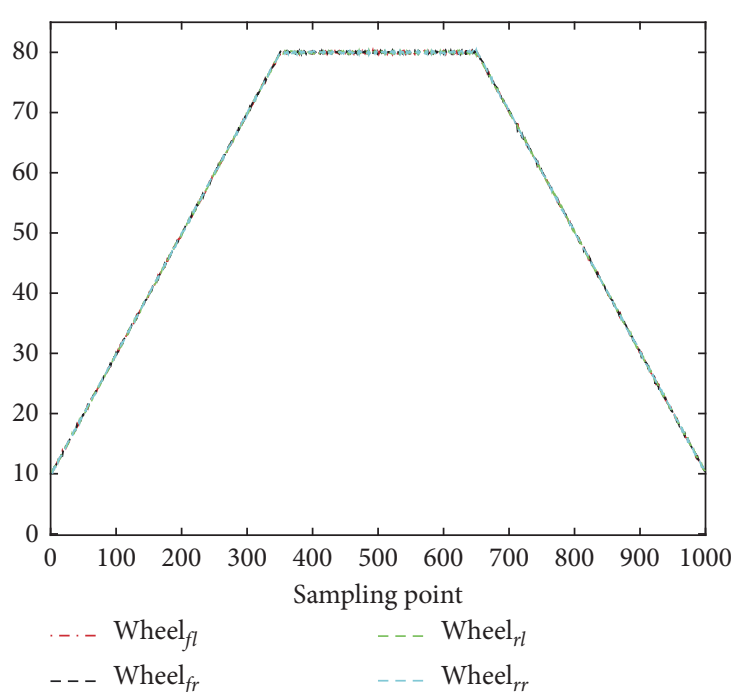

(b)

Figure 7: Continued. 

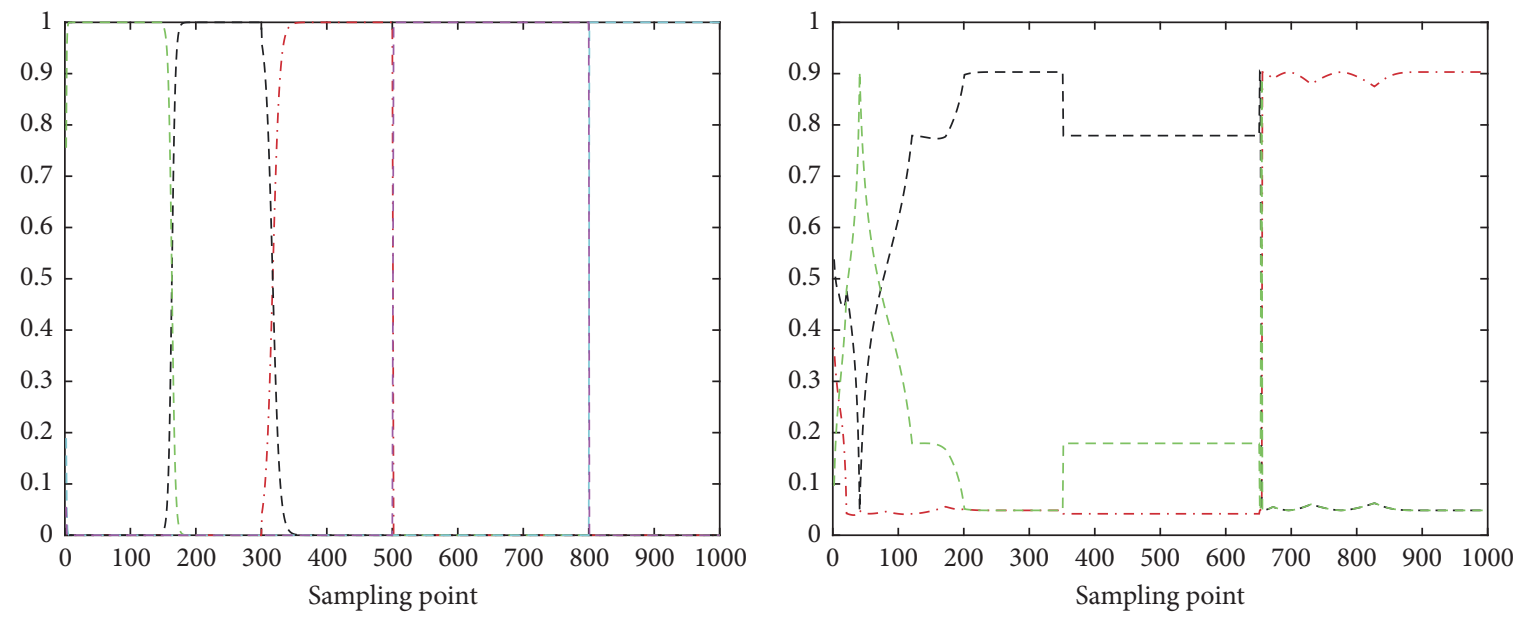

$$
\begin{array}{llll}
--\xi_{1} & --\xi_{4} & -. \lambda_{1} \\
---\xi_{2} & ---\xi_{5} & ---\lambda_{2} \\
---\xi_{3} & & ---\lambda_{3}
\end{array}
$$

(c)

(d)

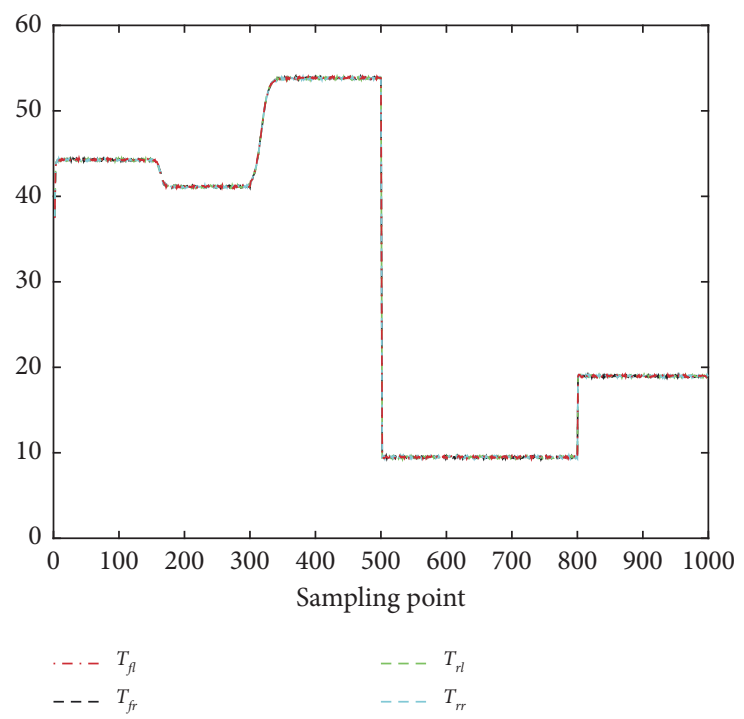

(e)

Figure 7: The simulation results of MPC-OSRCS on test 1. (a) Tire longitude slip ratio $\kappa$. (b) Vehicle velocity. (c) Weight of road surfaces. (d) Weight of operation modes. (e) Distribution of motor torque.

adhesion coefficient changes can be ignored. However, the slip ratio is affected by operation modes and road surfaces, and the optimal slip ratio control cannot be achieved under different operation modes and various road surfaces. Therefore, it is proved that the conventional MPC cannot be suitable for the multimodes and the conventional MPC cannot achieve optimal slip ratio control performance in actual working.

We can see from Figure 8(b) that the motor torques remain unchanged. In order to be suitable for different operation modes and various road surfaces, the motor torque should change, following the state of EV. However, the motor torque of four wheels $T_{e}(e=1,2,3,4)$ are appropriate and equal to $80 \mathrm{~N}$, whether the road surface is wet asphalt or snowy. Therefore, it is difficult to ensure the longitudinal stability of EV under traditional MPC, while EV works on various operation modes and different road surfaces. The conventional MPC cannot ensure driver comfort and longitudinal stability under this situation.

From the simulation of traditional MPC, we can conclude that it is difficult to realize the optimal slip ratio control and smooth switching, while EV works on various operation modes and different road surfaces.

4.2.2. The Performance of MPC-MMCS. The simulation results of MPC-MMCS are shown in Figure 9. 


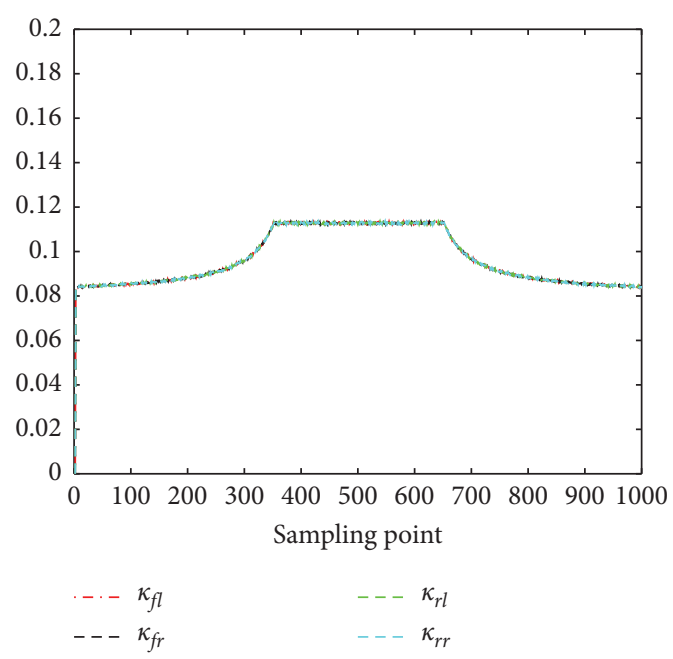

(a)

FIgURE 8: The simulation results of conventional MPC on test2. (a) Tire longitude slip ratio $\kappa$. (b) Distribution of motor torques.

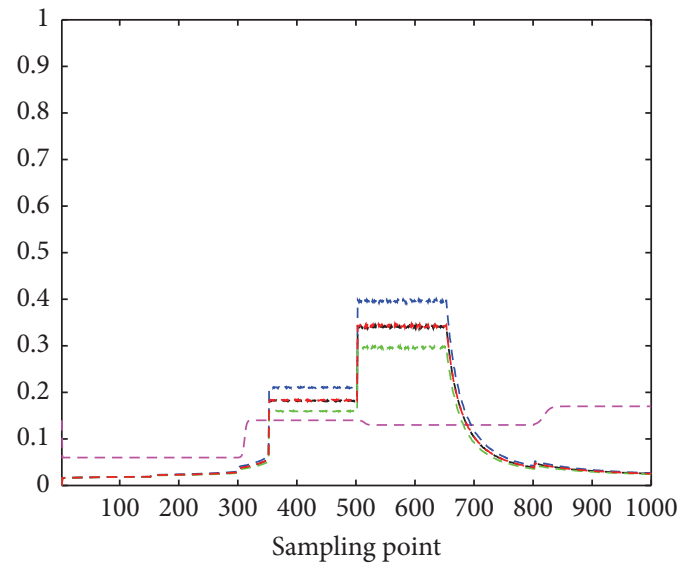

$$
\begin{array}{ll}
- & \kappa_{f l} \\
--- & \kappa_{f r} \\
--- & \kappa_{r l}
\end{array}
$$$$
\text { - - } \kappa_{r r}
$$$$
---\kappa_{o p}
$$

(a)

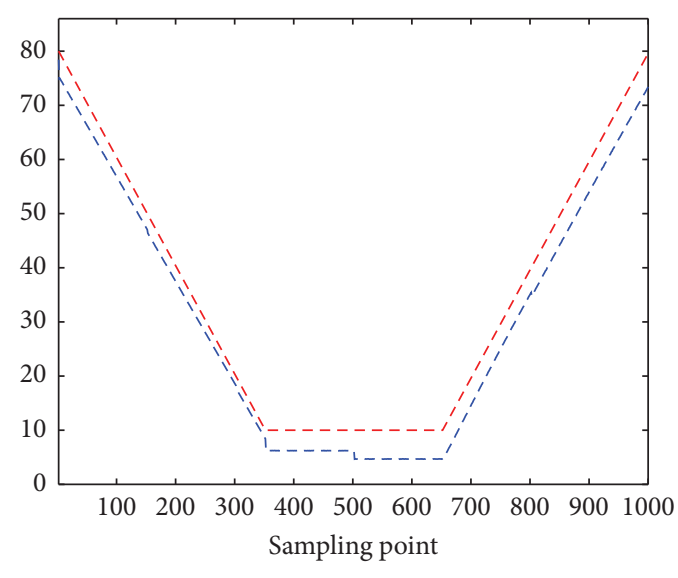

- - v

- . - Wheel

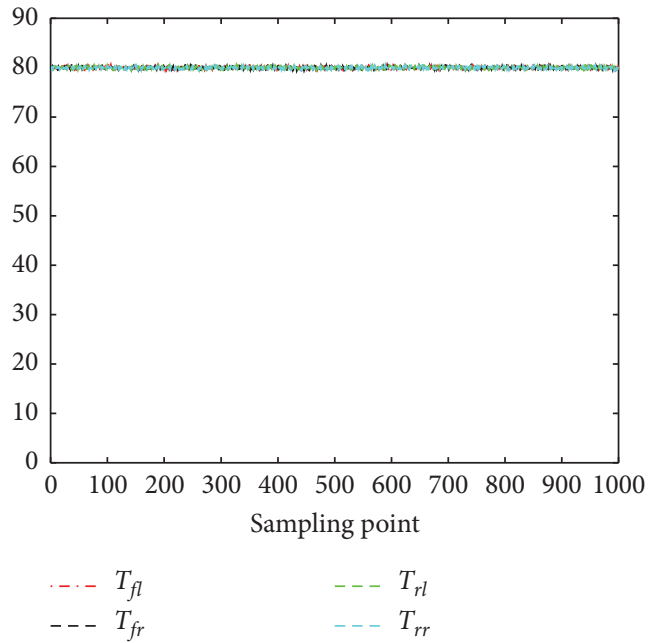

(b)

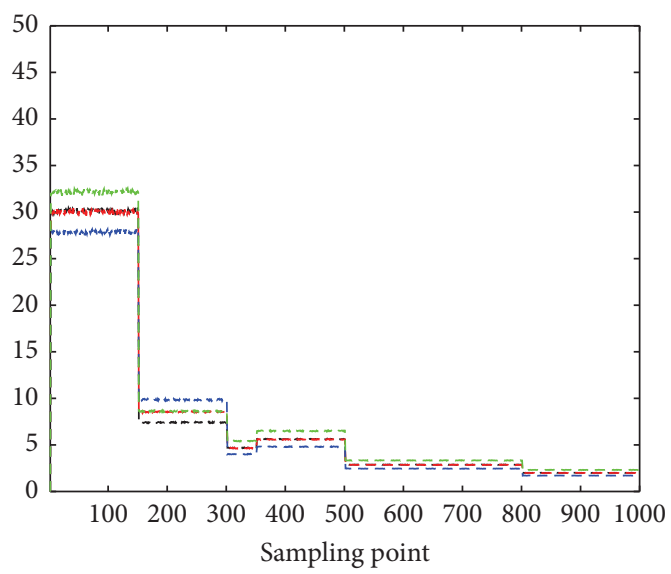

$$
\begin{array}{ll}
---T_{f l} & -- \\
---T_{f r} & T_{r l} \\
& ---T_{r r}
\end{array}
$$

(b)

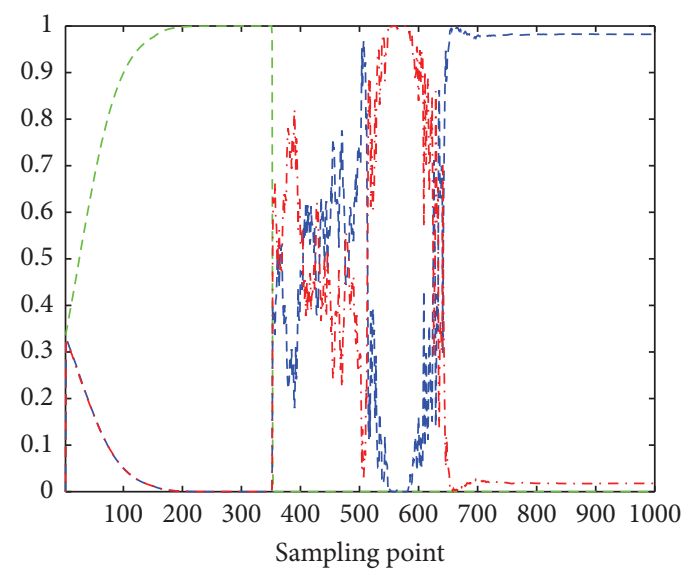

-..- Weight of model1

- - Weight of model2

-. Weight of model3

(d)

Figure 9: The simulation results of MPC-MMCS on test 2. (a) Tire longitude slip ratio $\kappa$. (b) Distribution of motor torques. (c) Vehicle velocity. (d) Weight of operation modes. 


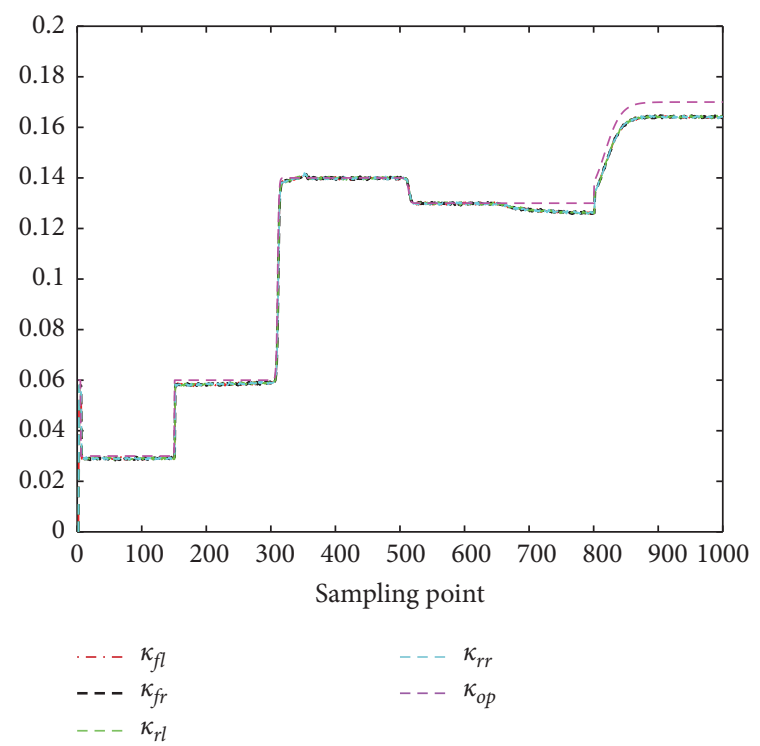

(a)

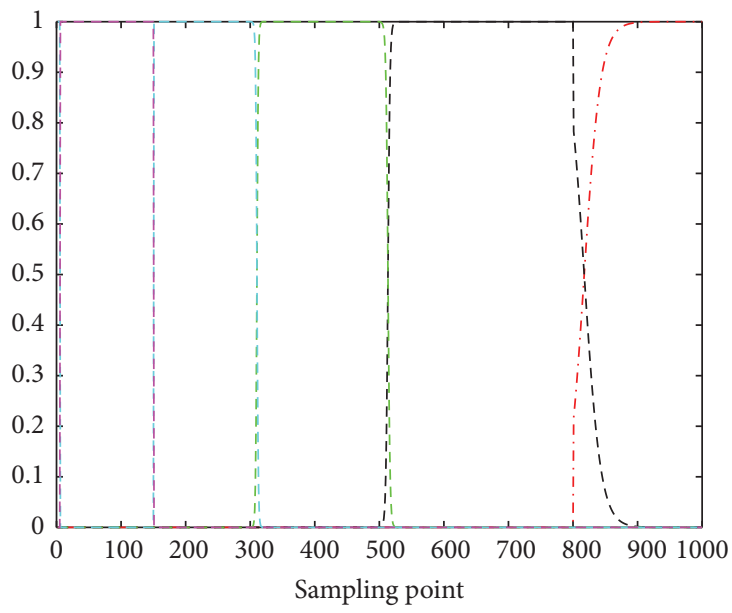

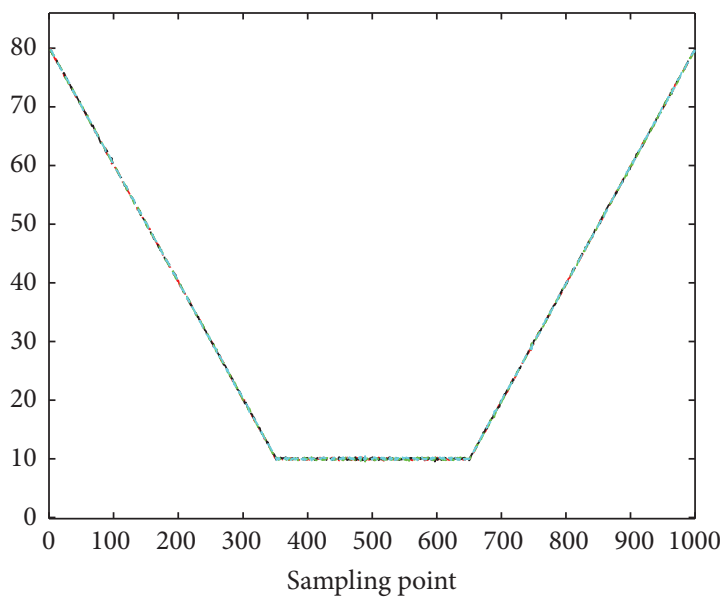

-..- Wheel $f l$

-. Wheel $\mathrm{H}_{r l}$

-.. Wheel $\mathrm{W}_{r r}$

(b)

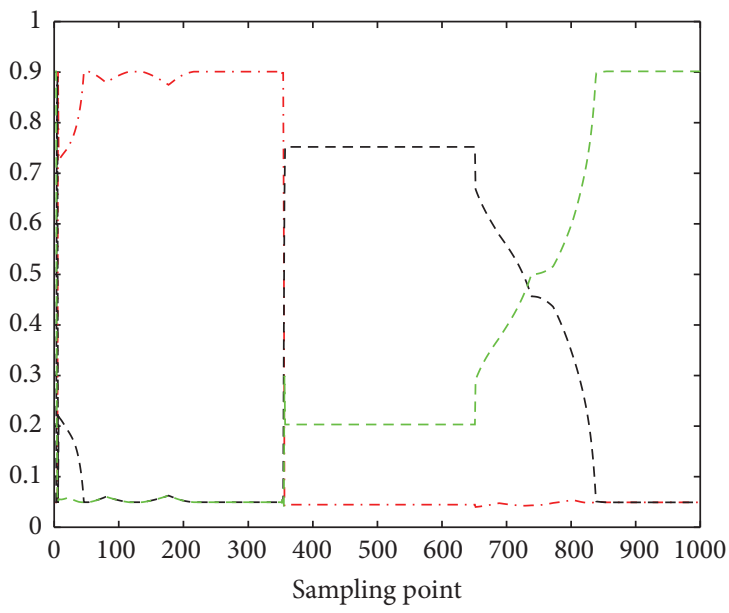

$$
\begin{array}{ll}
\ldots & \lambda_{1} \\
--- & \lambda_{2} \\
--- & \lambda_{3}
\end{array}
$$

(d)

(c)

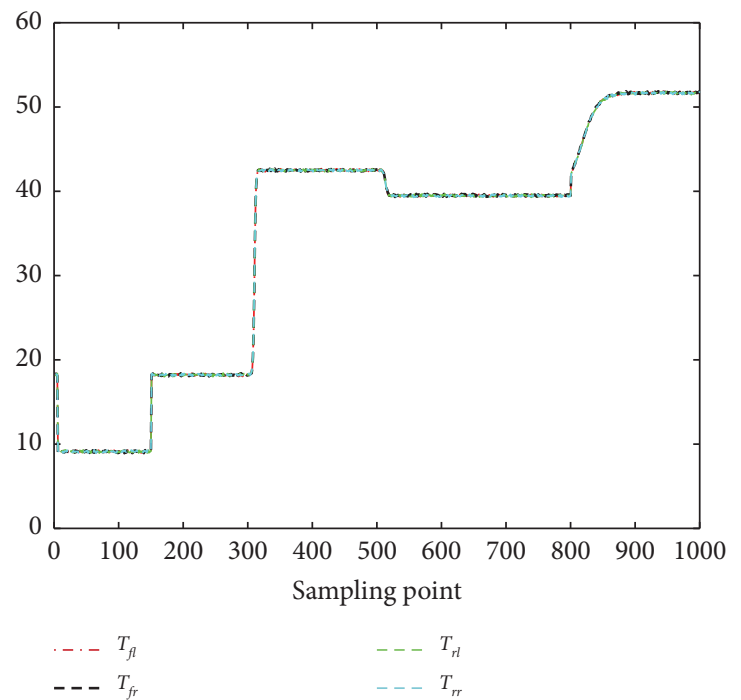

(e)

FIgURE 10: The simulation results of MPC-OSRCS on test 2. (a) Tire longitude slip ratio $\kappa$. (b) Vehicle velocity. (c) Weight of road surfaces. (d) Weight of operation modes. (e) Distribution of motor torques. 
As shown in Figure 9(a), the optimal slip rate follows the road surfaces change in electric vehicle operation. However, the optimal slip rate cannot be achieved when the road surfaces changes. It is easy to find in Figure 9(b) that $T$ is also kept within a stable range. The wheel velocity is displayed in Figure 9(c), and the smooth switching can be achieved when the EV works under different operation modes and various surfaces road surfaces. In Figure $9(\mathrm{~d})$, the $\lambda_{i}$ accurately reflects the operation modes of EV.

4.2.3. The Performance of MPC-OSRCS. The simulation results of the proposed MPC-OSRCS are shown in Figure 10.

We can see from Figure 10(a) the EV works under different road surfaces and various operation modes, and the slip ratio is controlled at the optimal slip ratio for the corresponding road surface, no matter how the state of EV changes. Compared with the traditional MPC, the slip ratio $\kappa$ changes quickly when the state of EV changes, and it can be proved that the rapidity of the controller is good. The slip ratio can be effectively achieved by the MPC-OSRCS and can be suitable for various operation modes and different road surfaces.

Figure 10(b) illustrates the test on wheel velocity $r \cdot \omega$. It is observed that EV runs smoothly under various operation modes and different roads surfaces. We can see from Figures $10(\mathrm{c})$ and $10(\mathrm{~d})$ that the identifier can quickly distinguish when the state of EV changes. As described above, the matching degree change following the state of EV changes and can precisely reflect the state of EV changes under different road surfaces and various operation modes.

We can see from Figure 10(e) that $T$ changes smoothly while the state of EV change, whether it is the change of operation modes or the road surfaces, the $T$ is also kept within a stable range $T=240$. The difference between motor torques of four wheels is small, and the comfortable of EV is well.

Compared with the conventional MPC, the proposed MPC-OSRCS can identify different operation modes and various road surfaces, and optimal slip ratio control can be realized. The simulation results reveal that the proposed MPC-OSRCS can better ensure the longitudinal stability of $\mathrm{EV}$ under different operation modes and various road surfaces.

\section{Conclusions}

Aiming at solving the problem that the traditional MPC cannot realize the optimal slip control while EV switches on various operation modes and different road surfaces, a novel MPC-OSRCS is proposed in this paper. It can not only identify different operation modes but also recognize various road surfaces. The control performance of MPC-OSRCS for $\mathrm{EV}$ is verified while EV works on different operation modes and various road surfaces. Simulation results demonstrate the advantage of MPC-OSRCS. Compared with the conventional MPC, the MPC-OSRCS can effectively improve longitudinal stability performance and achieve the optimal slip ratio control under various operation states and different road surfaces.

\section{Data Availability}

The raw/processed data required to reproduce these findings cannot be shared at this time as the data also forms part of an ongoing study.

\section{Conflicts of Interest}

The authors declare that they have no conflicts of interest.

\section{Acknowledgments}

This work was supported in part by the National Key R\&D Program of China (no. 2018YFF0212900).

\section{References}

[1] B. Gasbaoui and A. Nasri, "A novel 4WD electric vehicle control strategy based on direct torque control space vector modulation technique," Intelligent Control and Automation, vol. 3, no. 3, pp. 236-242, 2012.

[2] K. Jalali, T. Uchida, J. McPhee, and S. Lambert, "Development of a fuzzy slip control system for electric vehicles with inwheel motors," SAE International Journal of Alternative Powertrains, vol. 1, no. 1, pp. 46-64, 2012.

[3] F.-K. Wu, T.-J. Yeh, and C.-F. Huang, "Motor control and torque coordination of an electric vehicle actuated by two inwheel motors," Mechatronics, vol. 23, no. 1, pp. 46-60, 2013.

[4] W. Y. Wang, I. H. Li, M. C. Chen, and S. F. Su, "Dynamic slipratio estimation and control of antilock braking systems using an observer-based direct adaptive fuzzy-neural controller," IEEE Transactions on Industrial Electronics, vol. 56, no. 5, pp. 1746-1756, 2009.

[5] K. Shi, X. Yuan, G. Huang, and Q. He, "MPC-based compensation control system for the yaw stability of distributed drive electric vehicle," International Journal of Systems Science, vol. 49, no. 8, pp. 1795-1808, 2018.

[6] M. Yu and D. Wang, "Model-Based health monitoring for a vehicle steering system with multiple faults of unknown types," IEEE Transactions on Industrial Electronics, vol. 61, no. 7, pp. 3574-3586, 2014.

[7] L. Yuan, H. Zhao, H. Chen, and B. Ren, "Nonlinear MPCbased slip control for electric vehicles with vehicle safety constraints," Mechatronics, vol. 38, pp. 1-15, 2016.

[8] T. Nakakuki, T. Shen, and K. Tamura, "Adaptive control approach to uncertain longitudinal tire slip in traction control of vehicles," Asian Journal of Control, vol. 10, no. 4, pp. 67-73, 2008.

[9] W. Pasillas-Lépine, A. Loría, and M. Gerard, "Design and experimental validation of a nonlinear wheel slip control algorithm," Automatica, vol. 48, no. 8, pp. 1852-1859, 2012.

[10] V. M. Zavala and L. T. Biegler, "The advanced-step NMPC controller: optimality, stability and robustness," Automatica, vol. 45, no. 1, pp. 86-93, 2009.

[11] K. Shi, X. Yuan, and L. Liu, "Model predictive controllerbased multi-model control system for longitudinal stability of distributed drive electric vehicle," ISA Transactions, vol. 72, pp. 44-56, 2018. 
[12] X. Yuan, T. Zhang, Y. Xiang, and X. Dai, "Parallel chaos optimization algorithm with migration and merging operation," Applied Soft Computing, vol. 35, pp. 591-604, 2015.

[13] H. B. Pacejka, Tyre and Vehicle Dynamics, Elsevier, London, UK, 2nd edition, 2015.

[14] N. Ding and S. Taheri, "A modified dugoff tire model for combined-slip forces," Tire Science and Technology, vol. 38, no. 3, pp. 228-244, 2010.

[15] L. Gang, Z. Chang-fu, Z. Qiang, and H. Wei, "Acceleration slip regulation control of 4 WID electric vehicles based on fuzzy road identification," Journal of South China University of Technology, vol. 40, no. 12, pp. 99-104, 2012.

[16] D. Yin, N. Sun, D. Shan, and J.-S. Hu, "A multiple data fusion approach to wheel slip control for decentralized electric vehicles," Energies, vol. 10, no. 4, p. 461, 2017.

[17] N. Mutoh, "Driving and braking torque distribution methods for front-and rear-wheel-independent drive-type electric vehicles on roads with low friction coefficient," IEEE Transactions on Industrial Electronics, vol. 59, no. 10, pp. 39193933, 2012.

[18] B. Ren, H. Chen, H. Zhao, and L. Yuan, "MPC-based yaw stability control in in-wheel-motored EV via active front steering and motor torque distribution," Mechatronics, vol. 38, pp. 103-114, 2016.

[19] R. Zhang, R. Lu, A. Xue, and F. Gao, "New minmax linear quadratic fault-tolerant tracking control for batch processes," IEEE Transactions on Automatic Control, vol. 61, no. 10, pp. 3045-3051, 2016.

[20] B. Wang, R. Y. Sun, and Y. H. Xu, "Road surface condition identification approach with consideration of road roughness," Journal of Mechanical Engineering, vol. 48, no. 24, pp. 127-133, 2012.

[21] X. Sun, C. Yuan, Y. Cai, S. Wang, and L. Chen, "Model predictive control of an air suspension system with damping multi-mode switching damper based on hybrid model," Mechanical Systems and Signal Processing, vol. 94, pp. 94-110, 2017.

[22] N. Li, S.-Y. Li, and Y.-G. Xi, "Multi-model predictive control based on the Takagi-Sugeno fuzzy models: a case study," Information Sciences, vol. 165, no. 3-4, pp. 247-263, 2004.

[23] P. Di and L. Magni, "A multi-model structure for model predictive control," Annual Reviews in Control, vol. 28, no. 1, pp. 47-52, 2004.

[24] T. Bächle, K. Graichen, M. Buchholz, and K. Dietmayer, "Slipconstrained model predictive control allocation for an allwheel driven electric vehicle," IFAC Proceedings Volumes, vol. 47, no. 3, pp. 12042-12047, 2014.

[25] M. Morari and U. Maeder, "Nonlinear off set-free model predictive control," Automatica, vol. 45, no. 10, pp. 22142222, 2009. 